\title{
ESTUDIO DE LA VARIACIÓN DE LA COMPOSICIÓN QUÍMICA DEL ACEITE ESENCIAL DE LAS PARTES AÉREAS DE Nasa ranunculifolia (Kunth)
}

Arnold Jesús Pantoja Sánchez ${ }^{1}$, Otilia Acha de la Cruz ${ }^{2 \dagger}$, Ingrit Elida Collantes Díaz ${ }^{1,3 *}$

\section{RESUMEN}

El presente estudio tiene como objetivo investigar la influencia de la variación estacional sobre la composición química del aceite esencial de las partes aéreas de Nasa ranunculifolia, una planta efímera colectada en La Libertad (LLacuabamba) a una altura de $4052 \mathrm{msnm}$. Fueron identificados 36 compuestos de los cuales E- $\beta$-ionona, 2-Pentadecanona, fitol, n-octadecanol y los alcanos, n-tricosano, n-pentacosano, heptacosano y nonacosano son los que se mantienen presentes los cinco meses de colecta.

Palabras clave: Loasaceae, Nasa ranunculifolia, aceite esencial

\section{STUDY OF THE VARIATION OF THE CHEMICAL CONSTITUENTS OF THE ESSENTIAL OIL OF THE AERIAL PARTS FROM Nasa ranunculifolia (Kunth)}

\begin{abstract}
The study present aims to investigate the influence of seasonal variation on chemical composition of the essential oil of the aerial parts of Nasa ranunculifolia, an ephemeral plant collected in La Libertad (LLacuabamba) at $4502 \mathrm{msnm}$. Thirty six compounds were identified, of which E- $\beta$-ionone, 2-Pentadecanone, phytol, n-octadecanol and the alkanes, n-tricosane, n-pentacosane, heptacosane and nonacosano are those are present the five months of collection.
\end{abstract}

Key words: Loasaceae, Nasa ranunculifolia, essential oil

\section{INTRODUCCIÓN}

La familia Loasaceae, en el Perú, está representada por 8 géneros y 112 especies, siendo la

\footnotetext{
${ }^{1}$ Pregrado, Facultad de Ingeniería Química y Textil, Universidad Nacional de Ingeniería, Av. Túpac Amaru 210 Rímac, Lima-Perú, ingrit_uni@hotmail.com

${ }^{2}$ Laboratorio LABICER de la Facultad de Ciencias, Universidad Nacional de Ingeniería

${ }^{3}$ Postgrado, Facultad de Ciencias, Universidad Nacional de Ingeniería
} 
mayoría de ellas herbáceas y presentan pelos urticantes. El género Nasa pertenece a esta familia y es muy rica en especies que son encontradas en la región mesoandina, puna húmeda y seca y bosques muy húmedos montanos, desde 1400 hasta $4700 \mathrm{msnm}$. Nasa ranunculifolia es un arbusto efímero (noviembre - abril) y puede ser encontrada desde el sur de Cajamarca hasta La Libertad ${ }^{1}$, en la región norte del Perú es conocida bajo el nombre popular de ortiga colorada, ishanka de león y son usadas popularmente como antiinflamatorios ${ }^{2}$, por entrevistas con los locales indican que el uso como antiinflamario es frotándose la zona afectada con las partes aéreas de $N$. ranunculifolia.

Los aceites esenciales son mezclas complejas de compuestos volátiles, su presencia y abundancia puede verse afectada por varios factores, incluyendo el tiempo en la recolección, el estrés abiótico y bióticos. El estrés abiótico se debe principalmente a factores físicos y químicos. Condiciones como altas temperaturas, sequía, frío, alto contenido de sal, metales pesados y daño mecánico. El estrés biótico se debe principalmente a varios factores biológicos como hongos, bacterias, virus, nemátodos y plantas parasitarias ${ }^{3}$.

El objetivo del presente trabajo es el estudio, en términos de rendimiento y composición química, del aceite esencial de las partes aéreas de Nasa ranunculifolia colectadas durante cinco meses consecutivos en La Libertad (Llacuabamba). El aceite esencial fue analizado por cromatografía gaseosa acoplada a espectrómetro de masas y fueron identificados 36 componentes, de los cuales ocho compuestos están presentes en todos los meses colectados, E- $\beta$-ionona (norisopropanoide), 2-Pentadecanona (cetona alifática), fitol (diterpeno oxigenado), n-octadecanol (alcohol alifático) y cuatro alcanos, n-tricosano, n-pentacosano, heptacosano y nonacosano.

\section{PARTE EXPERIMENTAL}

Las partes aéreas de Nasa ranunculifolia (figura 1) fueron colectadas en La Libertad (Llacuabamba) a una altura de 4052 msnm (S 0803'37,29”, W 077²6’21,3”) durante 5 meses consecutivos, desde noviembre del 2019 hasta marzo del 2020. La identificación botánica fue realizada por el Museo de Historia Natural de la Universidad Nacional Mayor de San Marcos según el Sistema de Clasificación de Cronquist (1988) y fue clasificada como: perteneciente a la división Magnoliophyta, clase Magnoliopsida, subclase Dilleniidae, orden Violales, familia Loasaceae, género Nasa y especie Nasa ranunculifolia (Kunth) (figura 1).

Las masas de las partes aéreas colectadas en los meses de noviembre 2019, diciembre 2019, enero, febrero y marzo del 2020 fueron de 849,4 g, 874 g, 139 g, 303,1 g, 925 g, respectivamente. No se pudo colectar el mes de abril del 2020 debido a la cuarentena por la pandemia de la COVID 19. 


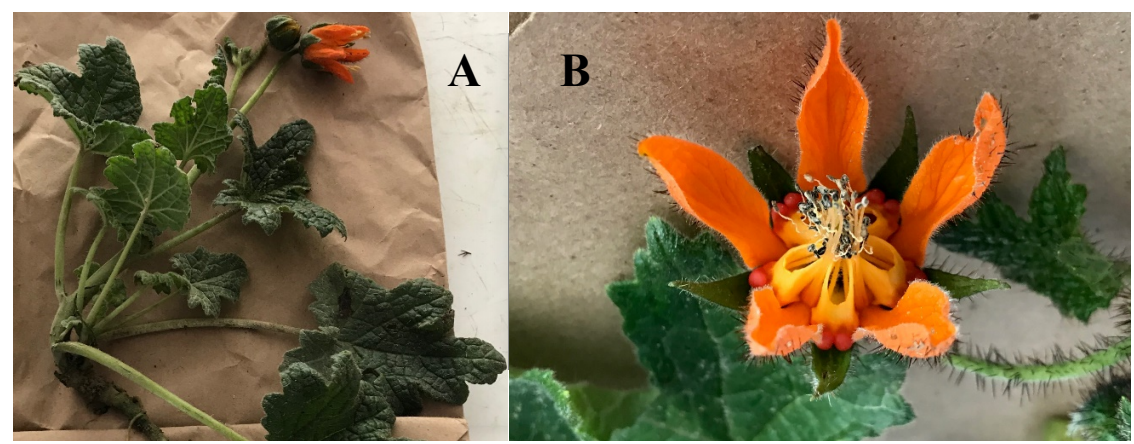

Figura 1. A: partes aéreas de Nasa ranunculifolia. B: flor de N. ranunculifolia (colectada en el mes de marzo del 2020).

\section{Extracción de los aceites esenciales}

Los aceites esenciales fueron obtenidos de las partes aéreas por hidrodestilación de 4 horas con aparato tipo Clevenger (Farmacopea), el aceite esencial fue extraído con éter de petróleo (EP) y secado con sulfato de sodio anhidro, el EP fue evaporado a temperatura ambiente y el aceite fue pesado para determinar su rendimiento y guardado en congeladora $\left(-20^{\circ} \mathrm{C}\right)$ hasta su análisis por cromatografía gaseosa acoplada a espectrometría de masas (CG-EM) ${ }^{4}$.

\section{Análisis de los aceites esenciales}

Las muestras del aceite esencial fueron preparadas a una concentración de $1 \mathrm{mg} / \mathrm{mL}$. El análisis por cromatografía gaseosa fue realizado en un cromatógrafo a gas acoplado a un espectrómetro de masas (Shimadzu, modelo CGMS-QP2010 Ultra) con las siguientes condiciones de análisis: columna capilar Restek Rtx-5MS (30 m x 0,25 mm x 0,25 $\mu \mathrm{m}$ ); temperatura del inyector $260{ }^{\circ} \mathrm{C}$, temperatura del detector $280{ }^{\circ} \mathrm{C}$; impacto de electrones a $70 \mathrm{eV}$, con gas de arrastre helio a un flujo de $1,48 \mathrm{~mL} / \mathrm{min}$, con split $1 / 5$; con un programa de temperatura de $80{ }^{\circ} \mathrm{C}\left(5^{\prime}\right)-280^{\circ} \mathrm{C}\left(5^{\prime}\right), 8{ }^{\circ} \mathrm{C} /$ minuto; y con volumen de inyección de $3 \mu \mathrm{L}$. La identificación de la composición química fue basada por la comparación de los espectros de masas con el banco de datos del sistema de CG-EM de NIST 14 lib y por análisis de sus espectros ${ }^{5}$.

\section{RESULTADOS Y DISCUSIÓN}

El aceite esencial obtenido a través de hidrodestilación de las partes aéreas de $N$. ranunculifolia fue transparente, el rendimiento de cada mes fue variado, como se observa en la tabla 1; los meses de diciembre y enero presentaron bajo rendimiento respecto a los otros meses debido a que las muestras estaban húmedas.

Fueron identificados un total de 36 compuestos en el aceite esencial (tabla 1), distribuidos en las siguientes categorías: Alcohol $(\mathbf{1}, \mathbf{6 , 7}, \mathbf{8 ,}, \mathbf{2 7}, \mathbf{2 9})$ alcanos alifáticos $(\mathbf{9}, \mathbf{3 0}, \mathbf{3 1}, \mathbf{3 2}$, 
$33,34,35,36)$ aldehídos $(2,5,10,11,13,17)$, ésteres (3) alquenos $(4)$, cetonas $(23,26)$, hidrocarburo monoterpénico $(12,14,15,16)$ monoterpenos oxigenados $(18,19,20,21,22)$, norisoprenoide (24), sesquiterpenos oxigenados (25), diterpenos oxigenados (28).

De los 36 compuestos identificados, los primeros 23 compuestos no son constantes en todos los meses, como el acetato de butilo (3), está en bajos porcentajes $(0,19 \%, 0,39 \%$ y 0,26 $\%$ ) en los meses de noviembre, febrero y marzo, este compuesto es ampliamente utilizado como materia prima para lacas, tintas, adhesivos, colorantes, solventes de pinturas y en la industria cosmética ${ }^{6}$; 2-hexenal (5) es comúnmente encontrada como componente volátil de plantas y se caracteriza porque es responsable del olor a hojas verdes y contribuye con la protección de la invasión de hongos e insectos ${ }^{7}$, este aldehído está presente en los meses de febrero $(0,7 \%)$ y marzo $(16,6 \%)$; el monoterpeno oxigenado linalool (18) que confiere aroma floral amaderado cítrico está presente, solamente, en los meses de diciembre, febrero y marzo en las proporciones de 3,09\%, 1,94 \% y 3,34 \% respectivamente, este monoterpeno es comúnmente encontrado en el aceite esencial de muchas plantas y presenta actividad antibacteriana, antiplasmódica, antiinflamatoria, antioxidante, anticancerígena, también es considerado como un atractivo para un amplio espectro de polinizadores (abejas, mariposas, etc.) y tiene efecto sobre el sistema nervioso central ${ }^{8}$.

Es a partir de los 46 minutos del cromatograma que se presentan compuestos constantes todos los meses como $\beta$-ionona, la cetona 2-Pentadecanona, el diterpeno oxigenado fitol, el alcohol alifático n-Octadecanol y los alcanos n-Tricosano, n-Pentacosano, heptacosano y nonacosano, como se presenta en la figura 2.

La $\beta$-ionona es un norisopropanoide cuya biosíntesis es a través del ácido mevalónico que se encuentra ampliamente distribuido en flores, hojas, cortezas, frutos y raíces de plantas $\mathrm{y}$ es comúnmente producido a bajos porcentajes en plantas ${ }^{9}$, este norisopropanoide es ampliamente obtenido como la fragancia de las violetas y es ampliamente usado en la industria de los aromas, incluyendo cosméticos y no-cosméticos ${ }^{10}$. En el presente estudio, fue identificado y está en bajos porcentajes $(1,12 \%, 2,61 \%, 1,8 \%, 1,15 \%$ y 1,25\%) durante los meses de colecta como afirma Paparella et al 2021. Presenta una amplia variedad de efectos farmacológicos como anticancerígeno, quimiopreventivo, antioxidante, antiinflamatorio y antimicrobial $^{10}$, regulador del crecimiento y de defensa de las plantas que producen ${ }^{9}$.

El diterpeno oxigenado fitol se encuentra como parte de la molécula de la clorofila y es producido por casi todos los organismos fotosintéticos como algas, plantas y algunas bacterias, es usado como constituyente de fragancias ya que es encontrado también en los aceites esenciales de algunas plantas ${ }^{11}$. En el aceite esencial de las partes aéreas de $N$. ranunculifolia está en las proporciones de $18,28 \%, 23,1 \%, 4,09 \%, 6,01 \%$ y 5,2\%, respectivamente, en cada mes de colecta; las muestras colectadas en los meses de noviembre y diciembre sólo presentaban hojas, a partir de la colecta de enero comenzó a aparecer las flores en botones, es probable que la presencia sólo de hojas en $N$. ranunculifolia es la razón por lo que los niveles de fitol sean altos $(18,28 \%$ y $23,1 \%)$ sólo en estos dos meses. El fitol presenta actividad ansiolítica, citotóxica, antioxidante, antiinflamatoria y antimicrobial ${ }^{11}$. 
El alcohol alifático 1-octadecanol está presente los 5 meses de colecta, este alcohol es utilizado en la producción de emulsiones, aceites textiles, antiespumante y lubricantes ${ }^{12}$.

Los alcanos n-Tricosano, n-Pentacosano, heptacosano y nonacosano son de cadena larga con número de carbonos impares, son los que están presentes en todos los meses de colecta. En la figura 2 se puede observar, en los meses de noviembre, diciembre, enero y marzo, la variación (\%) de los alcanos n-Tricosano, n-Pentacosano, heptacosano, mes a mes van de manera creciente, alcanzando siempre el máximo valor heptacosano; en estos mismos meses de colecta el nonacosano se mantiene prácticamente constante.

En el mes de febrero, se presentó una variación diferente ya que en este mes las proporciones van del $n$-Tricosano>n-Pentacosano>heptacosano>nonacosano $(13,57 \%, 12,59 \%, 7,68 \%$ y $2,42 \%$, respectivamente). Es en este mes que se presentan, solamente, los monoterpenos cis-Mirtanol y trans-Mirtanol en altos porcentajes 8,18 \% y 13,27 \%, respectivamente, ambos monoterpenos presentan actividad repelente contra los piojos ${ }^{13}$, también es reportado que el cis-Mirtanol presenta efecto sedante equivalente al pentobarbital ${ }^{14}$. 
Tabla 1. Porcentajes de rendimiento de los aceites esenciales de las partes aéreas de $N$. ranunculifolia colectados durante los meses de noviembre y diciembre del 2020 y enero, febrero y marzo del 2021; así como los componentes identificados, tiempo de retención.

\begin{tabular}{|c|c|c|c|c|c|c|c|}
\hline \multirow{3}{*}{$\mathbf{N}^{\circ}$} & & & \multirow{3}{*}{$\begin{array}{l}\text { Nov } \\
0,0034 \\
\end{array}$} & \multirow{2}{*}{$\begin{array}{l}\text { Dic } \\
0,0028 \\
\end{array}$} & \multirow{2}{*}{$\begin{array}{l}\text { Ene } \\
0,0023 \\
\end{array}$} & \multirow{2}{*}{$\begin{array}{l}\text { Feb } \\
0,0036 \\
\end{array}$} & \multirow{2}{*}{$\begin{array}{l}\text { Mar } \\
0,0034\end{array}$} \\
\hline & \multicolumn{2}{|l|}{ Porcentaje de rendimiento (w/w \%) } & & & & & \\
\hline & Nombre & TR & & & & & \\
\hline 1 & 2Z-Pentenol & 3,568 & - & - & - & - & 0,36 \\
\hline 2 & Hexanal & 4,187 & - & - & - & - & 1,59 \\
\hline 3 & Acetato de butilo & 4,635 & 0,19 & - & - & 0,39 & 0,26 \\
\hline 4 & 2E-Octene & 5,599 & - & - & - & - & 0,57 \\
\hline 5 & 2E-Hexenal & 5,772 & - & - & - & 0,7 & 16,6 \\
\hline 6 & 3Z-Hexenol & 5,953 & - & - & - & - & 5,33 \\
\hline 7 & 2Z-Hexenol & 6,322 & - & - & - & 0,55 & 1,27 \\
\hline 8 & n-Hexenol & 6,481 & - & - & - & - & 1,32 \\
\hline 9 & n-Nonano & 7,727 & - & - & - & - & 0,61 \\
\hline 10 & Heptanal & 7,848 & - & - & - & - & 0,61 \\
\hline 11 & Benzaldehído & 10,826 & - & - & - & - & 1,54 \\
\hline 12 & $\alpha$-Felandreno & 13,371 & - & - & - & - & 1,22 \\
\hline 13 & 2E,4E-Heptadienal & 13,877 & - & - & - & - & 1,69 \\
\hline 14 & p-Cimeno & 14,658 & - & - & - & - & 1,01 \\
\hline 15 & Silvestreno & 14,903 & - & - & - & - & 1,58 \\
\hline 16 & Z- $\beta$-Ocimeno & 15,691 & - & - & - & - & 0,73 \\
\hline 17 & Fenilacetaldehído & 15,937 & - & - & - & - & 0,94 \\
\hline 18 & Linalool & 20,282 & - & 3,09 & - & 1,94 & 3,34 \\
\hline 19 & Nopinona & 21,834 & - & - & - & 0,8 & - \\
\hline 20 & trans-Vervenol & 23,173 & 0,4 & - & - & - & - \\
\hline 21 & cis-Mirtanol & 29,747 & - & - & - & 8,18 & - \\
\hline 22 & trans-Mirtanol & 30,212 & - & - & - & 13,27 & - \\
\hline 23 & 3-Dodecanona & 43,391 & - & - & - & 1,57 & - \\
\hline 24 & $\beta$-ionona & 46,031 & 1,12 & 2,61 & 1,8 & 1,15 & 1,25 \\
\hline 25 & Espatulenol & 50,805 & 0,42 & - & - & - & \\
\hline 26 & 2-Pentadecanona & 65,804 & 2,2 & 2,8 & 0,72 & 2,46 & 1,17 \\
\hline 27 & n-Hexadecanol & 79,044 & - & - & 9,8 & - & \\
\hline 28 & Fitol & 80,363 & 18,28 & 23,1 & 4,09 & 6,01 & 5,2 \\
\hline 29 & n-Octadecanol & 85,955 & 3,01 & 10,16 & 5,75 & 4,54 & 1,18 \\
\hline 30 & n-Tricosano & 86,455 & 4,78 & 1,35 & 6,69 & 13,57 & 4,23 \\
\hline 31 & Tetracosano & 89,525 & 0,6 & - & - & 1,36 & 0,44 \\
\hline 32 & n-Pentacosano & 94,389 & 15,4 & 7,41 & 9,64 & 12,59 & 7,5 \\
\hline 33 & n-Hexacosano & 97,651 & 0,36 & - & - & - & - \\
\hline 34 & Heptacosano & 101,75 & 25,21 & 22,41 & 27,75 & 7,68 & 15 \\
\hline 35 & Octacosano & 104,43 & 0,56 & - & - & - & 1,28 \\
\hline 36 & Nonacosano & 108,62 & 12,98 & 12,98 & 12,68 & 2,42 & 14,24 \\
\hline & Alcohol alifático & & 3,01 & - & 15,55 & 0,55 & 9,43 \\
\hline & Alcano alifático & & 59,89 & 44,15 & 56,76 & 37,62 & 43,30 \\
\hline & Aldehído & & - & - & - & 0,7 & 22,03 \\
\hline & Ésteres & & 0,19 & - & - & 0,39 & 0,26 \\
\hline & Alquenos & & - & - & - & - & 0,57 \\
\hline & Cetonas & & 2,2 & 2,8 & 0,72 & 4,03 & 1,17 \\
\hline & Hidrocarburo Monoterpénico & & - & - & - & - & 4,54 \\
\hline & Monoterpenos oxigenados & & 0,4 & 3,09 & - & 24,19 & 3,34 \\
\hline & Norisoprenoide & & 1,12 & 2,61 & 1,8 & 1,15 & 1,25 \\
\hline & Sesquiterpeno oxigenado & & 0,42 & - & - & - & - \\
\hline & Diterpeno oxigenado & & 18,28 & 23,1 & 4,09 & 6,01 & 5,2 \\
\hline & Total de compuestos identificados (\%) & & 85,51 & 75,75 & 78,92 & 74,64 & 91,09 \\
\hline
\end{tabular}

TR: tiempo de retención, Nov: noviembre, Dic: diciembre, Ene: enero, Feb: febrero, Mar: marzo 


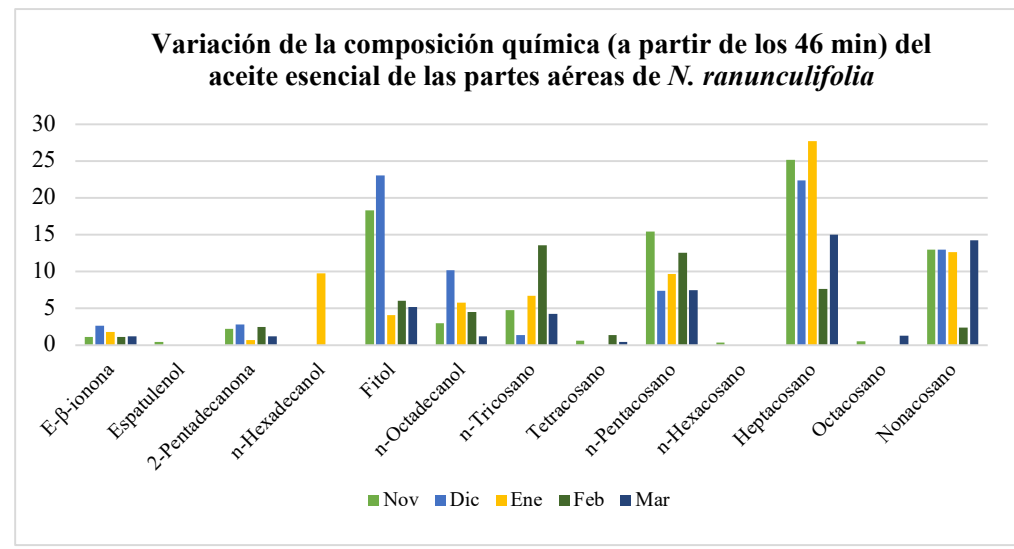

Figura 2. Variación estacional de la composición química del aceite esencial de las partes aéreas de $N$. ranunculifolia a partir de los $46 \mathrm{~min}$.

\section{CONCLUSIONES}

Con el presente trabajo se reporta, por primera vez, el estudio de la composición química del aceite esencial de las partes aéreas de $N$. ranunculifolia colectado por 5 meses consecutivos, en el que se observó que los 4 primeros meses la composición del aceite esencial no presenta hidrocarburos monoterpénicos, mientras que los monoterpenos oxigenados sólo no están presentes el mes de enero. Los compuestos observados constantemente presentes en todas las colectas fueron el norisoprenoide $\beta$-ionona, la cetona 2-Pentadecanona, el diterpeno oxigenado fitol, el alcohol n-Octadecano y los alcanos n-tricosano, n-Pentacosano, heptacosano y nonacosano.

\section{AGRADECIMIENTO}

Los autores agradecen al financiamiento del Vicerrectorado de Investigación de la Universidad Nacional de Ingeniería, Proyecto de Investigación Formativa FIQT-FI-31-2020.

\section{REFERENCIAS BIBLIOGRÁFICAS}

1. Rodríguez E, Weigend M. Loasaceae endémicas del Perú. Rev Peru Biol. 2006; 13(2): 391s-402s.

2. Rodríguez EFR, Izquierdo EA, Velásquez LP, Rodríguez EH, Cayatopa VR, Gallac SM, Pizarro YP. Estado actual de la flora con pelos urticantes en la región La Libertad, Perú. Sagasteguiana. 2014: 2(2): 113-134. 
3. Wang X, Li N, Li W, Gao X, Cha M, Qin L, Liu L. Advances in Transcriptomics in the Response to Stress in Plants. Glob Med Genet. 2020;7(2):30-34.

4. Oliveira TS, Bombo AB, Oliveira AS, Garcia VL, Appezzato-DA-Glória B. Seasonal variation of the essential oil from two Brazilian native Aldama La Llave (Asteraceae) species. An Acad Bras Cienc. 2016;88(3 Suppl):1899-1907.

5. Adams RP. Identification of essential oil components by gas chromatography/mass spectrometry. 4th Ed. Carol Stream, Illinois, USA: Allured Publishing Corporation; 2007.

6. Londoños AO, Lenis FL, Mendivelso LAB, Niño GR, Suárez LMC. Estudio de la síntesis de acetato de butilo y análisis de las condiciones de equilibrio. Ing. Investig. 2005; 25(3): 13-21.

7. Kunishima M, Yamauchi Y, Mizutani M, Kuse M, Takikawa H, Sugimoto Y. Identification of (Z)-3: (E)-2-Hexenal isomerases essential to the production of the leaf aldehyde in plants. J Biol Chem. 2016; 291(27): 14023-14033.

8. Kamatou GPP, Viljoen AM. Linalool - A review of a biologically active compound of commercial importance. Nat Prod Commun. 2008; 3(7): 1183-1192.

9. PaparellaA, Shaltiel-Harpaza L, Ibdah M. $\beta$-ionone: Its occurrence and biological function and metabolic engineering. Plants. 2021: 10(4):754. doi:10.3390/plants10040754.

10. Aloum L, Alefishat E, Adem A, Petroianu G. Ionone is more than a Violet's fragance: A Review. Molecules. 2020; 25(24):5822. doi.org/10.3390/molecules25245822.

11. Islam MT, Ali ES, Uddin ShJ, Ahmed MI, Shill MCh, Karmakar UK, et al. Phytol: A review of biomedical activities. Food Chem Toxicol. 2018;121:82-94.

12. The Merck Index, 12th ed. Boca Ratón, FL: CRC Press; 1996. Entry \# 8960.

13. Miyazawa M, Suzuki Y, Kameoka H. Biotransformation of (-)-cis-Myrtanol and (+)-trans-Myrtanol by plant pathogenic fungus, Glomerella cingulate. Phytochem. 1997: 45(5): 935-943.

14. De Sousa DP, Raphael E, Brocksom U, Brocksom TJ. Sedative effect of monoterpene alcohols in mice: a preliminary screening. Z Naturforsch C J Biosci. 2007;62(7-8):5636. 